\title{
Effect of magnetic field on quantum capacitance of the nanoobject
}

\author{
Lukiyanets B. A., Matulka D. V. \\ Lviv Polytechnic National University \\ 12 S. Bandera str., 79013, Lviv, Ukraine
}

(Received 2 December 2015)

\begin{abstract}
Quantum capacity of nanoplate in a quantizing magnetic field is studied. Investigation shows that quantum capacitance of nanoplate has step-like dependence on the Fermi level or potential bias This form is caused, practically, only by the dimensional quantized states. Landau quantization is manifested only at low temperatures as slight renormalization of steps without changing their sizes.
\end{abstract}

Keywords: quantum capacitance, nanoplate, magnetic quantizing

2000 MSC: $81 \mathrm{~V} 99$

UDC: 537.9:537.63

Recently, quantum capacitance is the subject of intense of fundamental and applied researches. For the first time the concept was introduced in the [1] studying the behavior of electrons in twodimensional structures. This and subsequent works showed that the effect is shown in low-dimensional structures and nanoobjects. One of the main reasons appearance of the quantum capacitance is the specificity of the electronic spectrum in such structures (see, f.ex., [2-6]). The discrete nature of the electronic spectrum in nanostructures is the cause of features of their capacitance other than in bulk crystals. The discrete nature of electronic spectrum in nanostructures creates capacitance, unlike the capacitance in bulk crystals. If we analyze the spectrum band of bulk crystal with orthogonal symmetry in the effective mass approximation (Fig. 1a), then the spectrum in nanoplate, cut out of the crystal, becomes a set of two-dimensional minizones (Fig. 1b).

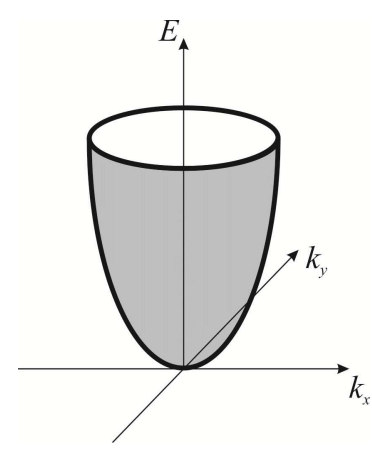

$a$

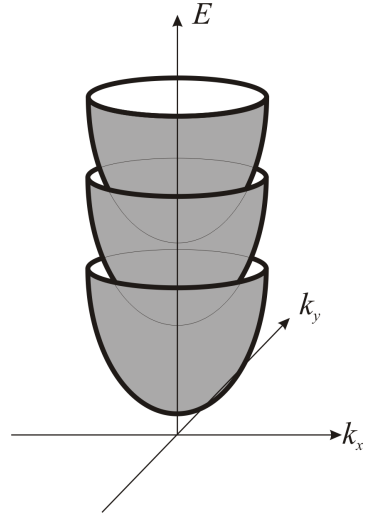

$b$

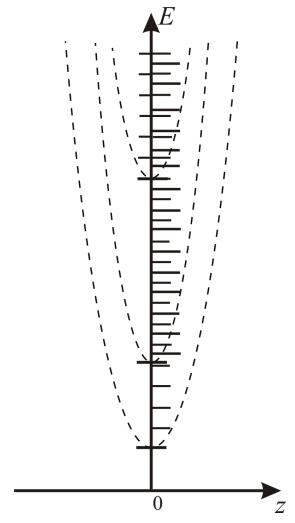

$c$

Fig. 1. Dispersion law of electrons in bulk crystal (a), in nanoplate, cut out of the crystal (b), electron states of the nanoplates in external magnetic field (c).

Applied magnetic field along the normal to the plate generates Landau quantization of each minibands (Fig. 1c). External quantizing magnetic field is widely used in the study in optical, magnetooptical properties of nanostructures [6-9]. Below we consider effect of magnetic field in quantum capacitance of nanoobjects. 


\section{Model}

The dispersion law of the carriers in semiconductors near the bottom of the conduction band or near the top of the valence band, and at low carrier concentrations in metal (see Fig. 1a) is well described by parabolic dependence:

$$
E_{\boldsymbol{k}}=\frac{\hbar^{2} k_{x}^{2}}{2 m_{x}}+\frac{\hbar^{2} k_{y}^{2}}{2 m_{y}}+\frac{\hbar^{2} k_{z}^{2}}{2 m_{z}}
$$

where $\boldsymbol{k}\left(k_{x}, k_{y}, k_{z}\right)$ is the quasimomentum, and $m_{x}^{*}, m_{y}^{*}, m_{z}^{*}$ are the effective masses along the corresponding axes. Further, consider a plate of the thickness $a$, cut out along $O Z$ of the crystal. When the potential, which limits plate, is an infinitely deep well, the solution of the stationary Schrödinger equation [10] takes form:

$$
E_{n}=\frac{\hbar^{2} \pi^{2}}{2 m_{z}^{*} a^{2}} n^{2}, \quad(n=1,2,3, \ldots)
$$

These are the stationary states with the more pronounced discreteness for the narrower width of the well. So in general, the dispersion law takes the form

$$
E_{n \boldsymbol{k}_{\|}}=\frac{\hbar^{2} k_{\|}^{2}}{2 m_{t}^{*}}+\frac{\hbar^{2} \pi^{2}}{2 m_{z}^{*} a^{2}} n^{2}
$$

(here we consider an isotropic in the $X O Y$ crystal plane with $m_{x}^{*}=m_{y}^{*}=m_{t}^{*} ; \boldsymbol{k}_{\|}\left(k_{x}, k_{y}\right), \boldsymbol{\rho}(x, y)$. Thus, the transition from the 3-dimensional crystal to the plate generates the minibands, i.e. the discrete states with the 2-dimensional bands in the plate plane. This is so-called quantum-size effect. Consider effects caused by the applied magnetic field $\boldsymbol{B}$ along $O Z$ of the 3-dimensional crystal. The behavior of an electron in the $X O Y$ plane is similar to the behavior of a harmonic oscillator that oscillates around the equilibrium position [11] with the energy

$$
E_{\nu}=\hbar \omega_{B}\left(\nu+\frac{1}{2}\right), \quad \nu=0,1,2, \ldots
$$

Here $\omega_{B}=\frac{e B}{c m_{t}^{*}}$ is cyclotron frequency.

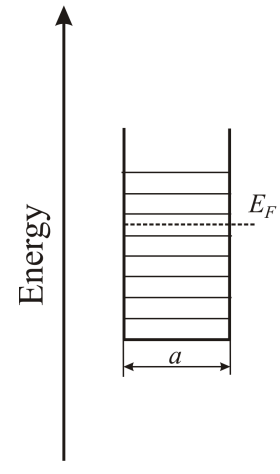

$a$

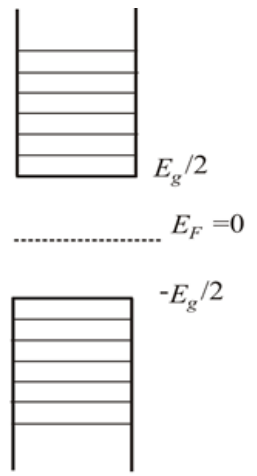

$b$

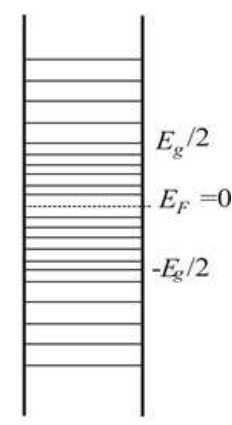

$c$

Fig. 2. Infinitely deep wells which simulate nanoplates: (a) metal; (b) semiconductor; (c) semimetal $\left(E_{F}\right.$ is the Fermi level, and $a$ is the width of the well).

In the case of an isotropic in the crystal plane $X O Y$ and uniform magnetic field $\boldsymbol{B}$ each quantum state $E_{\nu}$ is $N\left(\nu, k_{y}\right)$-multiple degenerate $N\left(\nu, k_{y}\right)=\frac{L_{x} L_{y} m_{t} \omega_{B}}{2 \pi \hbar}=\frac{L_{x} L_{y} e B}{2 \pi c \hbar} ; L_{x}, L_{y}$ are the crystal sizes in the $X O Y$ plane). For $k_{z}$ in the range $k_{z}, \cdots k_{z}+d k_{z}$, the total number of possible states per 
unit volume of the crystal is $N\left(\nu, k_{y}, k_{z}\right) d k_{z}=B \rho d k_{z}$ where $\rho=\frac{e}{4 \pi^{2} c \hbar}$ is the density of states. Thus, the magnetic field applied to plate ensures an additional to the size quantization discreteness of states:

$$
E_{v, k_{z}}=\hbar \omega_{B}\left(\nu+\frac{1}{2}\right)+\frac{\hbar^{2} \pi^{2}}{2 m_{l}^{*} a^{2}} n^{2}
$$

It is important to note that the discreteness of spectrum of the plate is uniquely determined by its size, while an applied magnetic field allows us to easily change such discreteness.

Use an infinitely deep rectangular well for simulation of low-dimensional plates of various electrical nature, namely metal, semiconductor, semimetal ones. Fig. 2 shows their potentials. Here we use the presented above electron energy states to analyze the quantum capacitance in such nanoplates.

\section{Calculation of quantum capacitance}

In the general case, the capacitance $C$ is defined as

$$
C=\frac{d Q}{d V}
$$

that is, a change of the charge $Q$ caused by the applied electrostatic field $V$. Here

$$
Q=e \sum_{n} f\left(E_{n}\right)
$$

where $e$ is a module of electron charge, and

$$
f\left(E_{n}\right)=\frac{1}{\exp \left(\frac{E_{n}-\mu+e V}{k T}\right)+1}
$$

is the Fermi-Dirac distribution ( $\mu$ is a chemical potential or Fermi level). The summation in (6) is over all quantum states of the system. It is easy to see that (1) can be represented alternatively as

$$
C=\frac{d Q}{d V} \Rightarrow-e \frac{d Q}{d \mu}
$$

Thus, $V$ and $\mu$ up to a factor identically determine the capacitance. In other words, the effect of its change can be realized by the potential shift, since the increase in $V$ shifts upward the zone and thus lifts $E_{F}$. The discrete nature of the electronic spectrum in nanostructures is the cause of features of their capacitance other than in bulk crystals. Before the calculations, let us make preliminary comments:

1. The necessary condition for experimental observation of the magnetic field effect is $\hbar \omega_{c} \gg k T$. As we consider the magnetic field in the region $B \in(0,10] \mathrm{T}$, even at its maximum value $\hbar \omega_{c} \approx 10^{-3}$ $\mathrm{eV}$, this corresponds to $T \approx 10 \mathrm{~K}$. Thus, manifestations of magnetic field can be expected at temperatures of about few units of Kelvin.

2. We will examine plate of the thickness of 8 and $10 \mathrm{~nm}$. In this case, the quantized levels in them, according to (4), are $\sim 4 \cdot 10^{-3} n^{2} \mathrm{eV}$ at $a=8 \mathrm{~nm}$ and $\sim 6 \cdot 10^{-3} n^{2} \mathrm{eV}$ at $a=10 \mathrm{~nm}$. 


\subsection{Metal nanoplate}

The expression for the quantum capacitance, taking into account formulas (3) and (4), in this case takes the following form:

$$
C_{q}=e \frac{d}{d V} \sum_{n} \frac{1}{\exp \left(\frac{E_{n}-\mu+e V}{k T}\right)+1}=-\frac{e^{2}}{4 k T} \sum_{n} \cosh ^{-2}\left(\frac{E_{n}-\mu+e V}{2 k T}\right)
$$

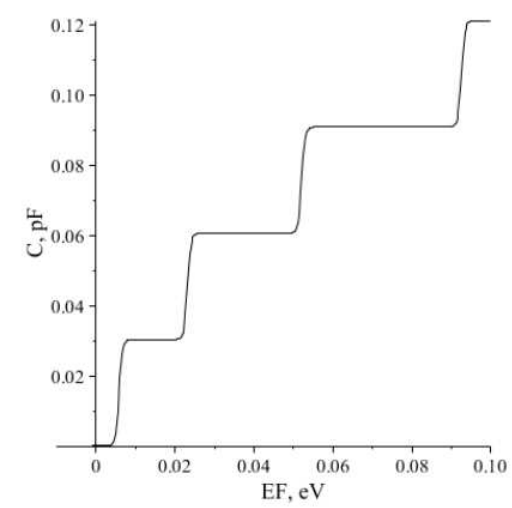

$a$

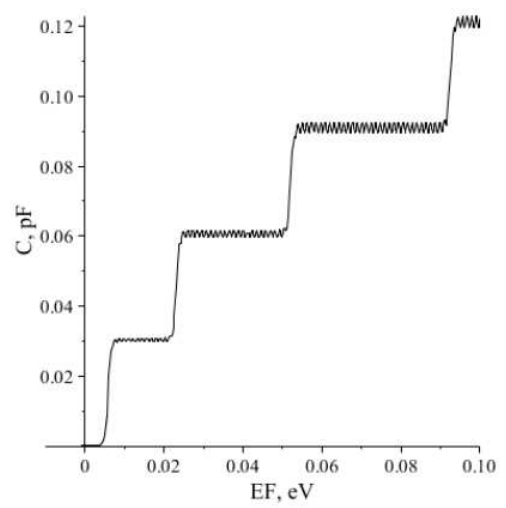

$b$

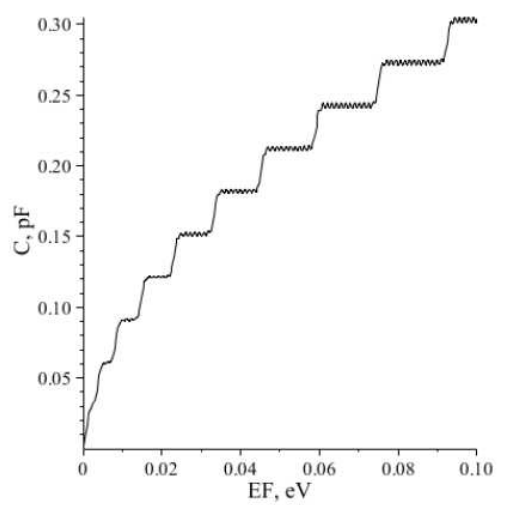

$c$

Fig. 3. Dependence of the quantum capacitance on the $E_{F}$ position in a metal nanoplate $\mathrm{nm}$ at $T=5 \mathrm{~K}$ in magnetic field: (a) $B=5 \mathrm{~T}$ with thickness $a=8 \mathrm{~nm}$; (b) $B=10 \mathrm{~T}$ with thickness $a=8 \mathrm{~nm}$; (c) $B=5 \mathrm{~T}$ with thickness $a=20 \mathrm{~nm}$. Bias voltage $V=0$.

The obtained dependence of quantum capacitance on the Fermi level position in a metal nanoplate with thickness $a=8 \mathrm{~nm}$ and at $T=5 \mathrm{~K}$ is presented in Fig. $3 a, 3 b$. It is seen that in both cases the curves have stair-like form. One can assume that their steps are the result of size quantization. The curve $C_{q}\left(E_{F}\right)$ at thickness $a=20 \mathrm{~nm}$ and at $B=10 \mathrm{~T}$ in Fig. $3 c$ confirms this assumption. Indeed, in this case, the distances between the size quantized states become shorter in comparison with those of the previous case (Fig. 3b), and hence, the length of the steps is reduced.

At $T=5 \mathrm{~K}$ (Fig. $3 b, 3 c)$ steps have periodically ordered structure; the higher the step is, the higher the density of its periodicity is. The cause of this phenomenon is existence of the Landau level. The described density is due to the fact that to the Landau levels of the $n$-th quantum-dimensional miniband the Landau levels of the $(n-1)$ lower minibands are added. It worth noting that with the exception of the ordered structure, observed at $B=10 \mathrm{~T}$ (Fig. $3 b$ ), the value of these steps and their position are the same as in the case of $B=5 \mathrm{~T}$ (Fig. $3 a$ ). The cause for the lack of manifestations of Landau levels at $B=5 \mathrm{~T}$ we discussed above. In particular, the curve $C_{q}(B)$ in Fig. 4 shows the significant dependence of its value on the magnetic field.

Calculations at higher temperatures indicate a fuzzy stair-like dependence of $C_{q}\left(E_{F}\right)$.

Fig. 5 shows that $C_{q}\left(E_{F}\right)$ substantial depends on temperature.

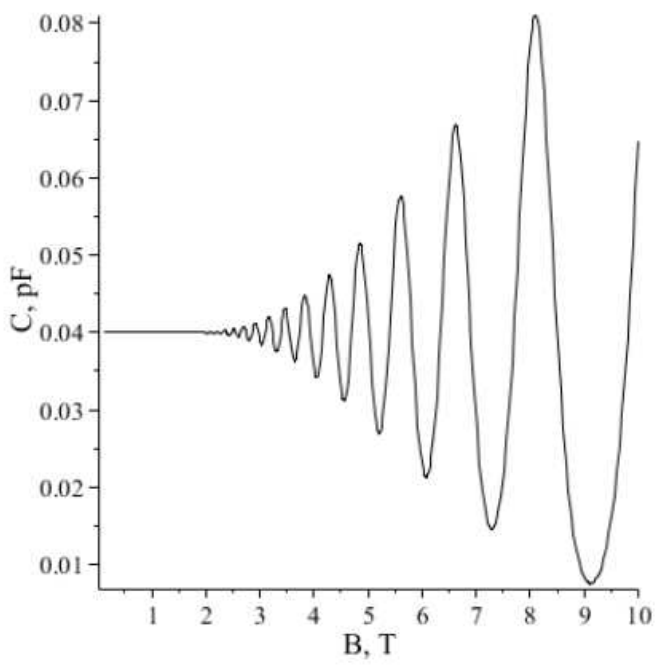

Fig. 4. Dependence of the quantum capacitance $C_{q}(B)$ of a metal nanoplate at a) $T=$ $=1 \mathrm{~K}, b) T=10 \mathrm{~K}$. Bias potential $V=0$. $E_{F}=0.01 \mathrm{eV} . a=8 \mathrm{~nm}$. 


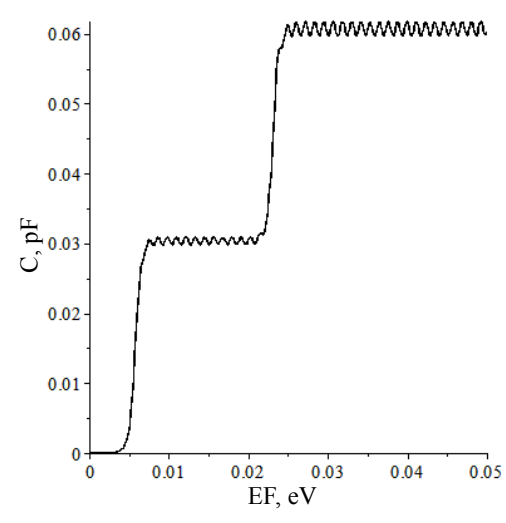

$a$

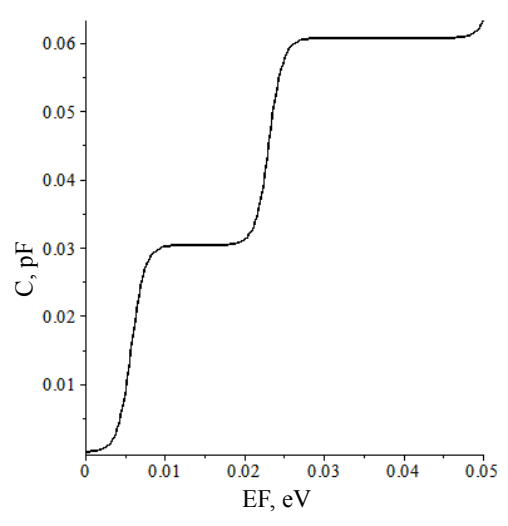

$b$

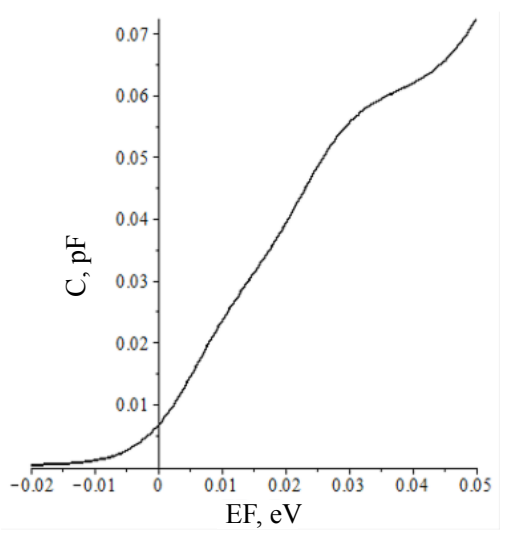

$c$

Fig. 5. Dependence of $C_{q}$ on $E_{F}$ at $B=10 \mathrm{~T}$ and: $T=5 \mathrm{~K}(a), T=10 \mathrm{~K}(b), T=50 \mathrm{~K}(c)$.

\subsection{Semiconductor nanoplate}

Consider a nanoplate of the semiconductor with symmetric conduction and valence bands (Fig. 1b). In this case, the Fermi level $E_{F}$ is in the middle of the band gap $E_{g}$. Choose this position as a datum point. Then charge caused by applied field is determined by the formula [11]:

$$
Q=e \sum_{s=1}^{\infty}\left\{f\left(E_{s}+\frac{E_{g}}{2}+e V\right)-f\left(E_{s}+\frac{E_{g}}{2}-e V\right)\right\}
$$

and the quantum capacitance by

$$
C_{q}=-\frac{e^{2}}{4 k T} \sum_{s=1}^{\infty}\left\{\cosh ^{-2}\left(\frac{E_{n}+E_{g} / 2+e V}{2 k T}\right)+\cosh ^{-2}\left(\frac{E_{n}+E_{g} / 2-e V}{2 k T}\right)\right\}
$$

As it is stated above, here summation is over all quantum states of the semiconductor nanoplate. Since the Fermi level is fixed in the middle of the band gap, the filling of states in both zones is due to the "tails" of the Fermi-Dirac distribution.

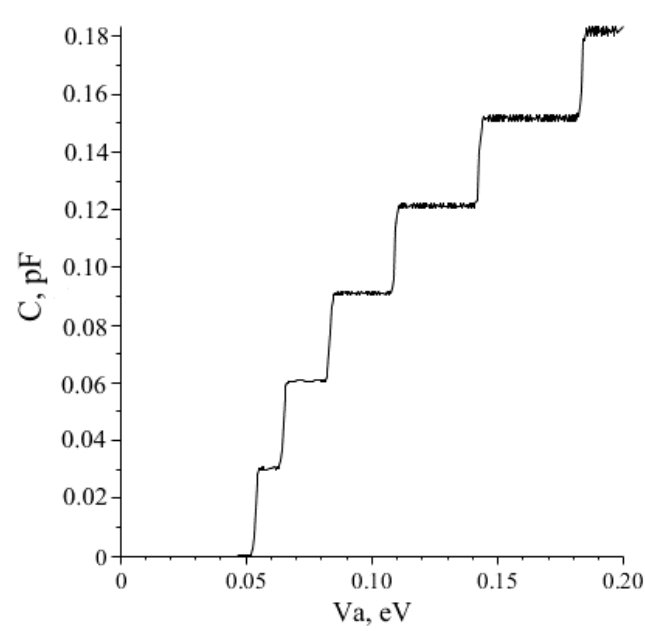

$a$

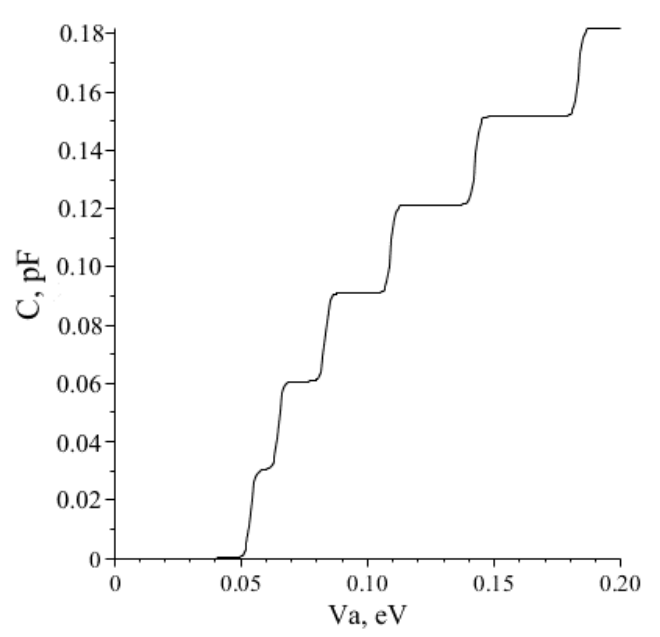

$b$

Fig. 6. Dependence of the quantum capacitance $C_{q}$ of a semiconductor nanoplate on the bias potential $V$ at $B=10 \mathrm{~T}$ at temperatures: $T=5 \mathrm{~K}(\mathrm{a}), T=10 \mathrm{~K}(\mathrm{~b}) a=10 \mathrm{~nm}$. 
Fig. 6 shows the quantum capacitance $C_{q}(V)$ in the magnetic field $B=10 \mathrm{~T}$ at different temperatures.

Comparison of these curves enables us to make the following conclusions:

The curves $C_{q}(V)$ are of stair-like form; they are is more fuzzy at higher temperature $(T=10 \mathrm{~K})$.

The nearly zero value of $C_{q}(V)$ starts to increase after $V=0.05 \mathrm{eV}$, i.e. when the Fermi level crosses the top of the valence band or the conduction band bottom (depending on the applied field).

Manifestations of the Landau levels are similar to those in metallic nanoplate.

\subsection{Semimetal nanoplate}

Quantum capacitance in this case can be analyzed using the formula (5) taking into account the fact that the conduction band bottom (valence band top) is $-E_{g} / 2\left(E_{g} / 2\right)$ (see Fig. 2c). Shown in Fig. 7

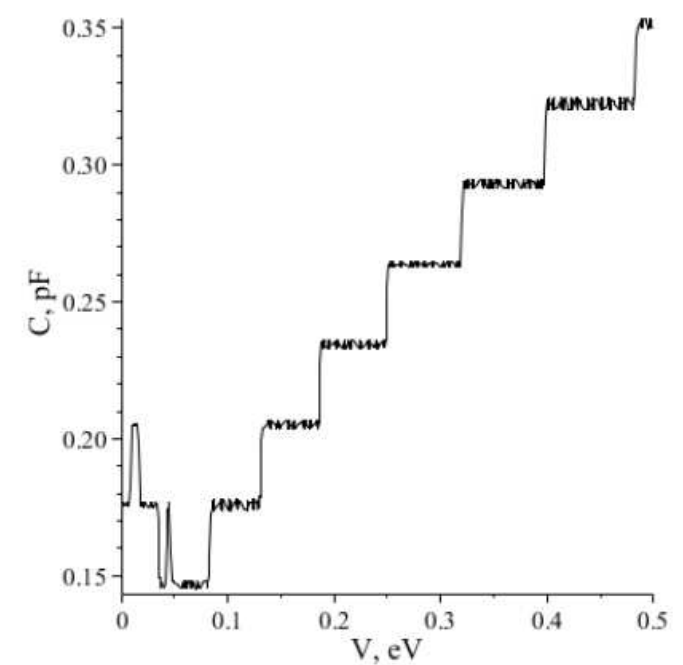

$a$

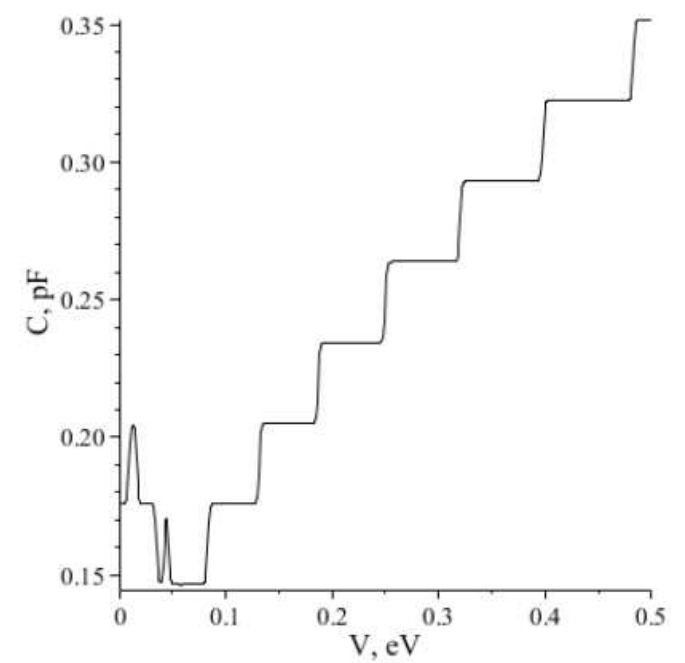

$b$

Fig. 7. Dependence of the quantum capacitance $C_{q}$ of a semimetal nanoplate on the bias potential $V$ in the magnetic field $B=10 \mathrm{~T}$ at the gap $E_{g}=-0.1 \mathrm{eV}$ and temperatures: (a) $T=5 \mathrm{~K}$ and (b) $T=10 \mathrm{~K} . a=10$ $\mathrm{nm}$.

dependence of quantum capacitance $C_{q}(V)$ at the overlapping zones $E_{g}=-0.1 \mathrm{eV}$ reminds one in previous cases, with the only exception of the range $V \in[0, \ldots 0.05] \mathrm{eV}$. Such nontrivial dependence is connected with the coexistence of electrons and holes in this range, and therefore their annihilation takes place. After the reaching $V=0.05$, when the bottom of the conduction band (or the top of the valence band) coincides with $E_{F}$, then stair-like form of $C_{q}(V)$ is the result of the dimension quantized states in the valence band (conduction band).

\section{Dicussion, conclusions}

We start with the choice of an isotropic crystal (metal, semiconductor, or semimetal) with a parabolic symmetric dispersion law. A nanoplate, which is cut-out from it, has minibands, i.e. a set of twodimensional zones at each quantum-dimensional discrete level. The magnetic field which is normally applied to the plate leads to additional quantization of band states also in its plane. In our case, it is a set of equally spaced levels (Landau levels).

Since the discreteness of spectrum is the one of the determining factors in the specific properties of nanoobjects, the application of a magnetic field is an additional factor of influence on these properties. Investigation of quantum capacitance of nanoplate as a function of the Fermi level position or potential bias has found its stair-like form; the lower the temperature is, the more pronounced such form is. This 
form is caused, practically, only by the size quantized states. Landau quantization is manifested only at low temperatures as slight renormalization of steps without changing their sizes.

[1] Luryi S. Quantum Capacitance Devices. Appl. Phys. Lett. 52, 501-503 (1988).

[2] John D. C., Castro L. C., Pulfrey D. L. Quantum Capacitance in Nanoscale Device Modelling. Appl. Phys. Lett. 96, 5180-51843 (2004).

[3] Dröscher, Roulleau P., Molitor F., Studerus P., Stampfer C., Ensslin K., Ihn T. Quantum capacitance and density of states of graphene Appl. Phys. Lett. 96, 152104 (2010).

[4] Xia J., Chen F., Li J., Tao N. Measurement of quantum capitance of graphene Nature Nanotechnology. 4, 505-509 (2009).

[5] Parkash V., Goel F. R. Quantum capacitance extraction for carbon nanotube interconnects Nanoscale Res. Lett. 5, 1424-1430 (2010).

[6] Zivanovic S., Milanovicl V., Ikovic Z. Intraband absorption in semiconductaing quantum wells in the presence of a perpendicular magnetic field Phys. Rev. B. 52, 8305-8311 (1995).

[7] Bacher G. Optical Spectroscopy on Epitaxially Grown II-VI Single Quantum Dots. Topics in Applied Physics. 90, 147-184 (2003).

[8] Salehani H. K., Zakeri M. Investigation of Light Absorption in a ZnS Quantum Dot. Journal of Spectroscopy. 2013, 39718-78911 (2013).

[9] Hakimyfard A., Barseghyan M. G., Kirakosyan A. A. Simultaneous effects of pressure and magnetic field on intersubband optical transitions in Pöschl-Teller quantum well. Physica E: Low-dimensional Systems and Nanostructures. 41, 1596-1599 (2009).

[10] Davydov A. S. Quantum Mechanics. Pergamon Press. (1965).

[11] Davydov A. S. Theory of Solids. Moscow, Nauka. (1980).

\title{
Вплив магнітного поля на квантову ємність нанооб'єктів
}

\author{
Лукіянець Б. А., Матулка Д. В. \\ Національний університет «Львівська політехніка» \\ вул. С. Бандери, 12, 79013, Львів, Україна
}

\begin{abstract}
Аналізується квантова ємність металічної, напівпровідникової та напівметалічної нанопластинок у квантуючому магнітному полі. Показано, що така ємність має сходинкову залежність від положення рівня Фермі чи потенціалу зміщення, що визначається майже винятково просторово-розмірними станами. Квантування Ландау проявляється за низьких температур як незначне перенормування сходинок без зміни їхніх розмірів.
\end{abstract}

Ключові слова: квантова ємність, нанопластинка, магнітне квантування 2000 MSC: $81 \mathrm{~V} 99$

УдК: 537.9:537.63 